\title{
General formal local cohomology modules
}

\author{
Sh. Rezaei
}

Communicated by V. Lyubashenko

A BSTRACT. Let $(R, \mathfrak{m})$ be a local ring, $\Phi$ a system of ideals of $R$ and $M$ a finitely generated $R$-module. In this paper, we define and study general formal local cohomology modules. We denote the $i$-th general formal local cohomology module $M$ with respect to $\Phi$ by $\mathfrak{F}_{\Phi}^{i}(M)$ and we investigate some finiteness and Artinianness properties of general formal local cohomology modules.

\section{Introduction}

Throughout this paper, $R$ is a commutative Noetherian ring with identity, $\mathfrak{a}$ is an ideal of $R, \Phi$ a system of ideals of $R$ and $M$ is an $R$ module. Recall that the $i$-th local cohomology module of $M$ with respect to $\mathfrak{a}$ is denoted by $\mathrm{H}_{\mathfrak{a}}^{i}(M)$. There are some generaliztions of local cohomology theory. The following one is given in [2]. A system of ideals of $R$ is a non-empty set $\Phi$ of ideals of $R$ such that, whenever $\mathfrak{a}, \mathfrak{b} \in \Phi$, there exists $\mathfrak{c} \in \Phi$ with $\mathfrak{c} \subseteq \mathfrak{a} \mathfrak{b}$. For such a system, there is a $\Phi$-torsion functor $\Gamma_{\Phi}: \mathcal{C}(R) \rightarrow \mathcal{C}(R)$ (where $\mathcal{C}(R)$ denotes the category of $R$-modules and $R$-homomorphisms) such that for every $R$-module $M$,

$$
\Gamma_{\Phi}(M):=\{x \in M: \mathfrak{a} x=0 \text { for some } \mathfrak{a} \text { in } \Phi\} .
$$

In [2], $\Gamma_{\Phi}(-)$ is called the "general local cohomology functor with respect to $\Phi^{\prime \prime}$. For each $i \geqslant 0$, the $i$-th right derived functor of $\Gamma_{\Phi}(-)$ is denoted by $\mathrm{H}_{\Phi}^{i}(-)$.

2010 MSC: 13D45, 13C14.

Key words and phrases: formal local cohomology, local cohomology, system of ideals. 
For more details about general local cohomology modules see [2], [3].

Let $\mathfrak{a}$ be an ideal of a local $\operatorname{ring}(R, \mathfrak{m})$ and $M$ a finitely generated $R$-module. For each $i \geqslant 0 ; \mathfrak{F}_{\mathfrak{a}}^{i}(M):=\lim _{n} \mathrm{H}_{\mathfrak{m}}^{i}\left(M / \mathfrak{a}^{n} M\right)$ is called the $\mathrm{i}$-th formal local cohomology of $M$ with respect to $\mathfrak{a}$.

The formal local cohomology modules have been studied by several authors; see for example [1], [4], [6], [9] and [10]. The purpose of this paper is to make a generalization of formal local cohomology theory as above. There are some generalization of formal local cohomology theory (see [7] and [11]). Here, we give a new generalization in terms of a system of ideals.

Let $(R, \mathfrak{m})$ be a local ring, $\Phi$ a system of ideals of $R$ and $M$ a finitely generated $R$-module. For each $i \geqslant 0$; we define $\mathrm{i}$-th general formal local cohomology of $M$ with respect to $\Phi$ by

$$
\mathfrak{F}_{\Phi}^{i}(M):=\lim _{\mathfrak{a} \in \Phi} \mathrm{H}_{\mathfrak{m}}^{i}(M / \mathfrak{a} M)
$$

Clearly, for an ideal $\mathfrak{a}$ of $R$, if we put $\Phi:=\left\{\mathfrak{a}^{i} \mid i \in \mathbb{N}\right\}$ then the above definition coincides with the original definition $\mathfrak{F}_{\mathfrak{a}}^{i}(M)$.

In this paper, we get some results on Artinianness, vanishing and other properties of general formal local cohomology modules. Among other things, we will prove that, for any finitely generated $R$-module $M$ we have:

$$
\begin{aligned}
\inf \{i & \left.\in \mathbb{N}: \mathfrak{F}_{\Phi}^{i}(M) \text { is not representable }\right\} \\
& =\inf \left\{i \in \mathbb{N}: \mathfrak{F}_{\Phi}^{i}(M) \text { is not Artinian }\right\}
\end{aligned}
$$

and

$$
\begin{aligned}
\sup \{i & \left.\in \mathbb{N}: \mathfrak{F}_{\Phi}^{i}(M) \text { is not representable }\right\} \\
& =\sup \left\{i \in \mathbb{N}: \mathfrak{F}_{\Phi}^{i}(M) \text { is not Artinian }\right\}
\end{aligned}
$$

Also, we study the structure of 0-th general formal local cohomology module and we will prove that for a complete local ring $(R, \mathfrak{m})$,

$$
\operatorname{Ass}_{R} \mathfrak{F}_{\Phi}^{0}(M)=\left\{\mathfrak{p} \in \operatorname{Ass}_{R}(M): \operatorname{dim} R /(\mathfrak{a}+\mathfrak{p})=0 \text { for all } \mathfrak{a} \in \Phi\right\}
$$

Recall that, $\operatorname{Assh}_{R}(M)$ denotes the set $\{\mathfrak{p} \in \operatorname{Ass} M: \operatorname{dim} R / \mathfrak{p}=\operatorname{dim} M\}$. We show that $\mathfrak{F}_{\Phi}^{\operatorname{dim} M}(M)$ is Artinian and there exists an ideal $\mathfrak{a}$ in $\Phi$ such that $\operatorname{Att}_{R} \mathfrak{F}_{\Phi}^{d}(M)=\operatorname{Assh}_{R}(M) \cap \mathrm{V}(\mathfrak{a})$. 


\section{Results}

Assume that $(R, \mathfrak{m})$ is a local ring and that $M$ is a finitely generated $R$-module. We investigate a generalization of formal local cohomology theory in terms of a system of ideals. A system of ideals of $R$ is a nonempty set $\Phi$ of ideals of $R$ such that, whenever $\mathfrak{a}, \mathfrak{b} \in \Phi$, there exists $\mathfrak{c} \in \Phi$ with $\mathfrak{c} \subseteq \mathfrak{a} \mathfrak{b}$. We define the relation $\leqslant$ on $\Phi$ by: $\mathfrak{a} \leqslant \mathfrak{b}$ if and only if $\mathfrak{b} \subseteq \mathfrak{a}$. It is easy to see that $\Phi$ is a direct set by this relation. Now, let $\mathfrak{a}, \mathfrak{b} \in \Phi$ such that $\mathfrak{a} \leqslant \mathfrak{b}, M$ be an $R$-module. Then for each integer $n \geqslant 0$, the $R$-homomorphism $M / \mathfrak{b} M \rightarrow M / \mathfrak{a} M$ induces the $R$-homomorphism $\psi_{\mathfrak{a}}^{\mathfrak{b}}: \mathrm{H}_{\mathfrak{m}}^{n}(M / \mathfrak{b} M) \rightarrow \mathrm{H}_{\mathfrak{m}}^{n}(M / \mathfrak{a} M)$. Thus $\left\{\mathrm{H}_{\mathfrak{m}}^{n}(M / \mathfrak{a} M), \psi\right\}$ forms an inverse system of $R$-modules and $R$-homomorphisms over $\Phi$. Now we are ready to give the following definition.

Definition 1. Let $(R, \mathfrak{m})$ be a local ring, $\Phi$ a system of ideals of $R$ and $M$ a finitely generated $R$-module. For each $i \geqslant 0 ; \mathfrak{F}_{\Phi}^{i}(M):=\lim _{\mathfrak{a} \in \Phi} \mathrm{H}_{\mathfrak{m}}^{i}(M / \mathfrak{a} M)$ is called the $\mathrm{i}$-th general formal local cohomology of $M$ with respect to $\Phi$.

Theorem 1. Let $(R, \mathfrak{m})$ be a local ring, $\Phi$ a system of ideals of $R$ and $M$ a finitely generated $R$-module. For each $i \geqslant 0 ; \mathfrak{F}_{\Phi}^{i}(M) \simeq \lim _{\mathfrak{a} \in \Phi} \mathfrak{F}_{\mathfrak{a}}^{i}(M)$.

Proof. Let $\mathfrak{a}, \mathfrak{b} \in \Phi$ such that $\mathfrak{a} \leqslant \mathfrak{b}$. If $n$ is an integer then the natural homomorphism $M / \mathfrak{b}^{n} M \rightarrow M / \mathfrak{a}^{n} M$ induces the homomorphism $\mathrm{H}_{\mathfrak{m}}^{i}\left(M / \mathfrak{b}^{n} M\right) \rightarrow \mathrm{H}_{\mathfrak{m}}^{i}\left(M / \mathfrak{a}^{n} M\right)$ for any integer $i \geqslant 0$. On the other hand, if $n \leqslant m$ we have the following commutative diagram:

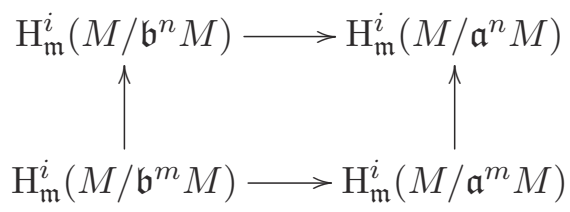

From the above diagram we get a homomorphism

$$
\lambda_{\mathfrak{a}}^{\mathfrak{b}}:{\underset{\swarrow}{n}}_{\lim } \mathrm{H}_{\mathfrak{m}}^{i}\left(M / \mathfrak{b}^{n} M\right) \rightarrow \underset{n}{\lim _{n}} \mathrm{H}_{\mathfrak{m}}^{i}\left(M / \mathfrak{b}^{n} M\right)
$$

and so, we have

$$
\lambda_{\mathfrak{a}}^{\mathfrak{b}}: \mathfrak{F}_{\mathfrak{b}}^{i}(M) \rightarrow \mathfrak{F}_{\mathfrak{a}}^{i}(M) .
$$

This shows that $\left\{\mathfrak{F}_{\mathfrak{a}}^{i}(M), \lambda\right\}_{\mathfrak{a} \in \Phi}$ is an inverse system of $R$-modules and $R$ homomorphisms over the directed set $\Phi$. Thus we may form $\lim _{\mathfrak{a} \in \Phi} \mathfrak{F}_{\mathfrak{a}}^{i}(-)$. But, for each integer $k \in \mathbb{N}$ and any ideal $\mathfrak{a} \in \Phi$ there exists an ideal $\mathfrak{b} \in \Phi$ such that $\mathfrak{b} \subseteq \mathfrak{a}^{k}$. Thus, by using a proof similar to the proof 
of [12, Lemma 3.8] for each integer $k$ we have

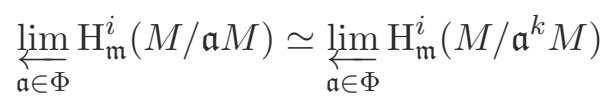

and so

$$
\begin{aligned}
& \lim _{\mathfrak{a} \in \Phi} \mathfrak{F}_{\mathfrak{a}}^{i}(M) \simeq \lim _{\mathfrak{a} \in \Phi} \lim _{k} H_{\mathfrak{m}}^{i}\left(M / \mathfrak{a}^{k} M\right) \simeq \varlimsup_{k} \lim _{\mathfrak{a} \in \Phi} \lim _{\mathfrak{a}} H_{\mathfrak{m}}^{i}\left(M / \mathfrak{a}^{k} M\right) \\
& \simeq \lim _{\mathfrak{a} \in \Phi} H_{\mathfrak{m}}^{i}(M / \mathfrak{a} M) \simeq \mathfrak{F}_{\Phi}^{i}(M)
\end{aligned}
$$

Let $(R, \mathfrak{m})$ be a local ring, $\Phi$ a system of ideals of $R$ and $M$ a finitely generated $R$-module. Let $\underline{x}$ denotes a system of elements of $R$ such that $\mathfrak{m}=\operatorname{Rad}(\underline{x} R)$. Let $\breve{C}_{\underline{x}}$ denotes the $\breve{C}$ ech complex of $R$ with respect to $\underline{x}$. For an $R$-module $M$ and an ideal $\mathfrak{a}$, it is easy to see that there exists an inverse system of $R$-complexes $\left\{\breve{C}_{\underline{x}} \otimes M / \mathfrak{a} M\right\}_{\mathfrak{a} \in \Phi}$. Hence, we may form the inverse limit $\lim _{\mathfrak{a} \in \Phi}\left(\breve{C}_{\underline{x}} \otimes M / \mathfrak{a} M\right)$. By a proof similar to the proof of [12, proposition 3.2] we obtain the next result.

Theorem 2. With the previous notation, there is an isomorphism

$$
\mathfrak{F}_{\Phi}^{i}(M) \simeq \mathrm{H}^{i}\left(\lim _{\mathfrak{a} \in \Phi}\left(\breve{C}_{\underline{x}} \otimes M / \mathfrak{a} M\right)\right)
$$

for all $i \in \mathbb{Z}$.

Proof. It follows by a straightforward modification of the proof of [12, proposition 3.2].

Theorem 3. Let $(R, \mathfrak{m})$ be a local ring, $\Phi$ a system of ideals of $R$ and $M$ a finitely generated $R$-module. Then $\mathfrak{F}_{\Phi}^{i}(M)=0$ for all $i>\operatorname{dim}(M)$.

Proof. Let $i>\operatorname{dim}(M)$. By [12, Theorem 4.5] $\mathfrak{F}_{\mathfrak{a}}^{i}(M)=0$ for all $\mathfrak{a} \in \Phi$. Thus $\mathfrak{F}_{\Phi}^{i}(M)=\lim _{\mathfrak{a} \in \Phi} \mathfrak{F}_{\mathfrak{a}}^{i}(M)=0$, as required.

Let $f: R \rightarrow R^{\prime}$ be a homomorphism of Noetherian commutative rings. Set $\Phi R^{\prime}:=\left\{\mathfrak{a} R^{\prime}: \mathfrak{a} \in \Phi\right\}$. Then $\Phi R^{\prime}$ is a system of ideals of $R^{\prime}$. Now by using this notation we give the following result:

Theorem 4. Let $(R, \mathfrak{m})$ be a local ring, $\Phi$ a system of ideals of $R$ and $M$ a finitely generated $R$-module. Then $\mathfrak{F}_{\Phi}^{i}(M) \simeq \mathfrak{F}_{\Phi \widehat{R}}^{i}(\widehat{M})$ for all $i \in \mathbb{Z}$.

Proof. By [12, Proposition 3.3], $\mathfrak{F}_{\mathfrak{a}}^{i}(M) \simeq \mathfrak{F}_{\mathfrak{a} \widehat{R}}^{i}(\widehat{M})$. Thus $\lim _{\mathfrak{a} \in \Phi} \mathfrak{F}_{\mathfrak{a}}^{i}(M) \simeq$ $\lim _{\mathfrak{a} \in \Phi} \mathfrak{F}_{\mathfrak{a} \widehat{R}}^{i}(\widehat{M})$. Now Theorem 1 completes the proof. 
Recall that a dualizing complex $D_{R}$ for a local ring $(R, \mathfrak{m})$ is a bounded complex of injective $R$-modules whose cohomology modules $\mathrm{H}^{i}\left(D_{R}^{i}\right)$ are finitely generated $R$-modules for all $i \in \mathbb{Z}$. For more details see [13]. It is well known that $R$ possesses a dualizing complex if and only if $R$ is the factor ring of a Gorenstein ring. The next result is an expression of the general formal local cohomology in terms of a certain general local cohomology of the dualizing complex.

Theorem 5. Let $(R, \mathfrak{m})$ be a local ring possessing a dualizing complex $D_{R}$, $\Phi$ a system of ideals of $R$ and $M$ a finitely generated $R$-module. Then

$$
\mathfrak{F}_{\Phi}^{i}(M) \simeq \operatorname{Hom}_{R}\left(\mathrm{H}_{\Phi}^{-i}\left(\operatorname{Hom}_{R}\left(M, D_{R}^{\cdot}\right)\right), E(R / \mathfrak{m})\right),
$$

for all $i \in \mathbb{Z}$.

Proof. By Local Duality Theorem there are the isomorphisms

$$
\mathrm{H}_{\mathfrak{m}}^{i}(M / \mathfrak{a} M) \simeq \operatorname{Hom}_{R}\left(\mathrm{H}^{-i}\left(\operatorname{Hom}_{R}\left(M / \mathfrak{a} M, D_{R}^{*}\right)\right), E(R / \mathfrak{m})\right),
$$

for all $i \in \mathbb{Z}$ and $\mathfrak{a} \in \Phi$. Thus we have

$$
\left.\lim _{\mathfrak{a} \in \Phi} \mathrm{H}_{\mathfrak{m}}^{i}(M / \mathfrak{a} M) \simeq \operatorname{Hom}_{R}\left(\mathrm{H}^{-i} \underset{\mathfrak{a} \in \Phi}{\lim _{\overparen{T}}} \operatorname{Hom}_{R}\left(M / \mathfrak{a} M, D_{R}^{*}\right)\right), E(R / \mathfrak{m})\right),
$$

for all $i \in \mathbb{Z}$. But $\left.\underset{\mathfrak{a} \in \Phi}{\lim } \operatorname{Hom}_{R}\left(M / \mathfrak{a} M, D_{R}^{*}\right)\right) \simeq \Gamma_{\Phi}\left(\operatorname{Hom}_{R}\left(M, D_{R}^{*}\right)\right)$ and so

$$
\varliminf_{\mathfrak{a} \in \Phi} H_{\mathfrak{m}}^{i}(M / \mathfrak{a} M) \simeq \operatorname{Hom}_{R}\left(\mathrm{H}^{-i}\left(\Gamma_{\Phi}\left(\operatorname{Hom}_{R}\left(M, D_{R}^{\cdot}\right)\right), E(R / \mathfrak{m})\right),\right.
$$

for all $i \in \mathbb{Z}$. Therefore

$$
\mathfrak{F}_{\Phi}^{i}(M) \simeq \operatorname{Hom}_{R}\left(\mathrm{H}_{\Phi}^{-i}\left(\operatorname{Hom}_{R}\left(M, D_{R}^{*}\right)\right), E(R / \mathfrak{m})\right),
$$

for all $i \in \mathbb{Z}$, as required.

Theorem 6. Let $(R, \mathfrak{m})$ be a local ring, $\Phi$ a system of ideals of $R$ and $0 \rightarrow A \rightarrow B \rightarrow C \rightarrow 0$ a short exact sequence of finitely generated $R$-modules. Then there is a long exact sequence

$$
\cdots \rightarrow \mathfrak{F}_{\Phi}^{i}(A) \rightarrow \mathfrak{F}_{\Phi}^{i}(B) \rightarrow \mathfrak{F}_{\Phi}^{i}(C) \rightarrow \mathfrak{F}_{\Phi}^{i+1}(A) \rightarrow \cdots .
$$

Proof. It follows by an argument similar to the proof of [12, Theorem 3.11]. 
Theorem 7. Let $(R, \mathfrak{m})$ be a local ring, $\Phi$ a system of $i d e a l s$ of $R$ and $M$ a finitely generated $R$-module. If $w:=\max \{\operatorname{dim}(M / \mathfrak{a} M) \mid \mathfrak{a} \in \Phi\}$ is finite then $\mathfrak{F}_{\Phi}^{w}(M) \neq 0$ and $\mathfrak{F}_{\Phi}^{i}(M)=0$ for all $i>w$.

Proof. Let $i>w$. Since $i>\operatorname{dim}(M / \mathfrak{a} M)$ for all $\mathfrak{a} \in \Phi$, [12, Theorem 4.5] implies that $\mathfrak{F}_{\mathfrak{a}}^{i}(M)=0$ for all $\mathfrak{a} \in \Phi$. Thus $\mathfrak{F}_{\Phi}^{i}(M)=\lim _{\mathfrak{a} \in \Phi} \mathfrak{F}_{\mathfrak{a}}^{i}(M)=0$. On the other hand, since $w$ is finite there exists an ideal $\mathfrak{b} \in \Phi$ such that $\operatorname{dim}(M / \mathfrak{b} M)=w$. Now, put $\Psi=\{\mathfrak{c} \in \Phi \mid \mathfrak{c} \subseteq \mathfrak{b}\}$. Then $\Psi$ is cofinal in $\Phi$. Thus we may assume that $\mathfrak{a} \subseteq \mathfrak{b}$ for all $\mathfrak{a} \in \Phi$. Let $\mathfrak{c} \in \Phi$. It is easy to see that $\operatorname{dim}(\mathfrak{b} M / \mathfrak{c} M) \leqslant \operatorname{dim} M / \mathfrak{c} M \leqslant w$ and so the exact sequence $0 \rightarrow \mathfrak{b} M / \mathfrak{c} M \rightarrow M / \mathfrak{c} M \rightarrow M / \mathfrak{b} M \rightarrow 0$ induces $\mathrm{H}_{\mathfrak{m}}^{w}(M / \mathfrak{c} M) \rightarrow$ $\mathrm{H}_{\mathfrak{m}}^{w}(M / \mathfrak{b} M) \rightarrow 0$. Now for each $\mathfrak{d} \in \Phi$ with $\mathfrak{d} \leqslant \mathfrak{c}$ i.e $\mathfrak{c} \subseteq \mathfrak{d}$ we have the following commutative diagram:

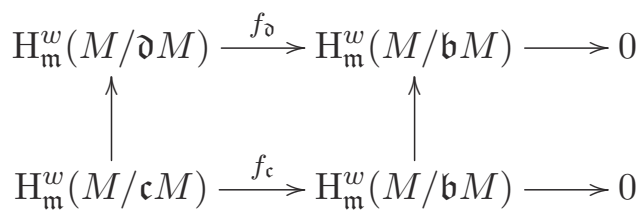

The family of $R$-modules $\left\{\operatorname{ker} f_{\mathfrak{c}}\right\}_{\mathfrak{c} \in \Phi}$, as a family of Artinian $R$-modules, satisfies the Mittag-Leffler condition. Hence the above diagram induces an exact sequence $\varliminf_{\mathfrak{c} \in \Phi} \mathrm{H}_{\mathfrak{m}}^{w}(M / \mathfrak{c} M) \rightarrow \mathrm{H}_{\mathfrak{m}}^{w}(M / \mathfrak{b} M) \rightarrow 0$. By Theorem 1 we get $\mathfrak{F}_{\Phi}^{w}(M) \rightarrow \mathrm{H}_{\mathfrak{m}}^{w}(M / \mathfrak{b} M) \rightarrow 0$. By Grothendieck's non-vanishing Theorem $\mathrm{H}_{\mathfrak{m}}^{w}(M / \mathfrak{b} M) \neq 0$. Therefore $\mathfrak{F}_{\Phi}^{w}(M) \neq 0$, as required.

Theorem 8. Let $(R, \mathfrak{m})$ be a local ring, $\Phi$ a system of ideals of $R$ and $M a$ finitely generated $R$-module of dimension $d$. Then $\mathfrak{F}_{\Phi}^{d}(M)$ is homomorphic image of $\mathrm{H}_{\mathfrak{m}}^{d}(M)$, and so $\mathfrak{F}_{\Phi}^{d}(M)$ is Artinian.

Proof. Let $\mathfrak{a} \in \Phi$. We have $\operatorname{dim} \mathfrak{a} M \leqslant \operatorname{dim} M$, so that, by the Grothendieck's Vanishing Theorem, the short exact sequence

$$
0 \longrightarrow \mathfrak{a} M \longrightarrow M \longrightarrow M / \mathfrak{a} M \longrightarrow 0
$$

induces an exact sequence

$$
\mathrm{H}_{\mathfrak{m}}^{d}(M) \stackrel{\phi_{\mathfrak{a}}}{\longrightarrow} \mathrm{H}_{\mathfrak{m}}^{d}(M / \mathfrak{a} M) \longrightarrow 0 .
$$

The family of $R$-modules $\left\{\operatorname{ker} \phi_{\mathfrak{a}}\right\}_{\mathfrak{a} \in \Phi}$, as a family of Artinian $R$-modules, satisfies the Mittag-Leffler condition. Therefore, the above exact sequence induces an exact sequence $\lim _{\mathfrak{a} \in \Phi} \mathrm{H}_{\mathfrak{m}}^{d}(M) \rightarrow \lim _{\mathfrak{a} \in \Phi} \mathrm{H}_{\mathfrak{m}}^{d}(M / \mathfrak{a} M) \rightarrow 0$ and 
so we have the exact sequence $\mathrm{H}_{\mathfrak{m}}^{d}(M) \rightarrow \mathfrak{F}_{\Phi}^{d}(M) \rightarrow 0$, and the proof is complete.

In the next result, we investigate the 0-th general formal local cohomology module. Let $\mathfrak{a}$ be an ideal of $R$ and $M$ a finitely generated $R$-module. For a submodule $N$ of $M$ we denote the ultimate constant value of the increasing sequence

$$
N \subseteq N: M \mathfrak{a} \subseteq N: M \mathfrak{a}^{2} \subseteq \cdots \subseteq N::_{M} \mathfrak{a}^{i} \subseteq \cdots
$$

by $N:_{M}\langle\mathfrak{a}\rangle$. Let $0=\bigcap_{j=1}^{n} Q_{j}$ denotes a reduced primary decomposition of the zero submodule 0 in $M$ and $Q_{j}$ is a $\mathfrak{p}_{j}$-primary submodule of $M$, for all $j=1, \cdots, n$. Put $T(\mathfrak{a}, M):=\left\{\mathfrak{p} \in \operatorname{Ass}_{R} M: \operatorname{dim} R /(\mathfrak{a}+\mathfrak{p})>0\right\}$ and $u_{M}(\mathfrak{a}):=\bigcap_{\mathfrak{p}_{i} \in T(\mathfrak{a}, M)} Q_{i}$ also $T(\Phi, M):=\left\{\mathfrak{p} \in \operatorname{Ass}_{R} M\right.$ : there exists $\mathfrak{a} \in$ $\Phi$ such that $\operatorname{dim} R /(\mathfrak{a}+\mathfrak{p})>0\}$ and $u_{M}(\Phi):=\bigcap_{\mathfrak{p}_{i} \in T(\Phi, M)} Q_{i}$. With these notations we have:

Theorem 9. Let $(R, \mathfrak{m})$ be a local ring, $\Phi$ a system of ideals of $R$ and $M$ a finitely generated $R$-module. Then

i) $\bigcap_{\mathfrak{a} \in \Phi} u_{M}(\mathfrak{a})=u_{M}(\Phi)$,

ii) $u_{M}(\Phi)=\bigcap_{\mathfrak{a} \in \Phi}(\mathfrak{a} M: M\langle\mathfrak{m}\rangle)$,

iii) $\mathfrak{F}_{\Phi}^{0}(M) \simeq u_{\hat{M}}(\Phi \hat{R})$.

Proof. i) It is easy to see that

$$
\bigcap_{\mathfrak{a} \in \Phi} u_{M}(\mathfrak{a})=\bigcap_{\mathfrak{a} \in \Phi} \bigcap_{\mathfrak{p}_{i} \in T(\mathfrak{a}, M)} Q_{i}=\bigcap_{\mathfrak{p}_{i} \in T(\Phi, M)} Q_{i}=u_{M}(\Phi) .
$$

ii) By [12, Lemma 4.1(a)], $u_{M}(\mathfrak{a})=\bigcap_{n \geqslant 1}\left(\mathfrak{a}^{n} M:_{M}\langle\mathfrak{m}\rangle\right)$. Thus

$$
u_{M}(\Phi)=\bigcap_{\mathfrak{a} \in \Phi} u_{M}(\mathfrak{a})=\bigcap_{\mathfrak{a} \in \Phi} \bigcap_{n \geqslant 1}\left(\mathfrak{a}^{n} M:_{M}\langle\mathfrak{m}\rangle\right) \subseteq \bigcap_{\mathfrak{a} \in \Phi}(\mathfrak{a} M: M\langle\mathfrak{m}\rangle) .
$$

Conversly, let $x \in \bigcap_{\mathfrak{a} \in \Phi}\left(\mathfrak{a} M:_{M}\langle\mathfrak{m}\rangle\right)$. Let $\mathfrak{a} \in \Phi$ be an ideal. Then there exists an integer $u$ such that $x \mathfrak{m}^{u} \subseteq \mathfrak{a} M$. For any integer $k$, there exists an ideal $\mathfrak{b} \in \Phi$ such that $\mathfrak{b} \subseteq \mathfrak{a}^{k}$. Since $x \in(\mathfrak{b} M: M\langle\mathfrak{m}\rangle)$ there exists an integer $t$ such that $x \mathfrak{m}^{t} \subseteq \mathfrak{b} M \subseteq \mathfrak{a}^{k} M$. Hence $x \in\left(\mathfrak{a}^{k} M:_{M}\langle\mathfrak{m}\rangle\right)$ and so $x \in \bigcap_{n \geqslant 1}\left(\mathfrak{a}^{n} M: M\langle\mathfrak{m}\rangle\right)$ for each ideal $\mathfrak{a} \in \Phi$. Therefore $x \in$ $\bigcap_{\mathfrak{a} \in \Phi} \bigcap_{n \geqslant 1}\left(\mathfrak{a}^{n} M:_{M}\langle\mathfrak{m}\rangle\right)=u_{M}(\Phi)$.

iii) By Theorem 4 we may assume that $M=\hat{M}$ and $R=\hat{R}$. Let $b$ be a proper ideal of $R$ such that $\mathfrak{b} \in \Phi$. It is easy to see that $\bigcap_{\mathfrak{a} \in \Phi} \mathfrak{a} M \subseteq$ $\bigcap_{n \geqslant 0} \mathfrak{b}^{n} M$. Thus Krull's intersection theorem implies that $\bigcap_{\mathfrak{a} \in \Phi} \mathfrak{a} M=0$. Now the proof is a straightforward modification of the proof of [12, Lemma $4.1(\mathrm{c})]$. 
Corollary 1. Let $(R, \mathfrak{m})$ be a complete local ring, $\Phi$ a system of ideals of $R$ and $M$ a finitely generated $R$-module. Then

$$
\operatorname{Ass}_{R} \mathfrak{F}_{\Phi}^{0}(M)=\left\{\mathfrak{p} \in \operatorname{Ass}_{R} M: \operatorname{dim} R /(\mathfrak{a}+\mathfrak{p})=0 \text { for all } \mathfrak{a} \in \Phi\right\}
$$

Proof. By [12, Lemma 2.7] $\operatorname{Ass}_{R} u_{M}(\Phi)=\operatorname{Ass}_{R} M \backslash T(\Phi, M)$. But

$$
\operatorname{Ass}_{R} M \backslash T(\Phi, M)=\left\{\mathfrak{p} \in \operatorname{Ass}_{R} M: \operatorname{dim} R /(\mathfrak{a}+\mathfrak{p})=0 \text { for all } \mathfrak{a} \in \Phi\right\}
$$

and $\mathfrak{F}_{\Phi}^{0}(M)=u_{M}(\Phi)$ by Theorem $9($ iii) and this finishes the proof.

Corollary 2. Let $(R, \mathfrak{m})$ be a local ring, $\Phi$ a system of ideals of $R$ and $M$ a finitely generated $R$-module. Then $\mathfrak{F}_{\Phi}^{0}(M)=0$ if and only if $\operatorname{Ass}_{\hat{R}} \hat{M}=$ $T(\Phi \hat{R}, \hat{M})$.

Proof. By Theorem 3(iii) $\mathfrak{F}_{\Phi}^{0}(M)=0$ if and only if $u_{\hat{M}}(\Phi \hat{R})=0$. But $\operatorname{Ass}_{\hat{R}} u_{\hat{M}}(\Phi \hat{R})=\operatorname{Ass}_{\hat{R}} \hat{M} \backslash T(\Phi \hat{R}, \hat{M})$ by [12, Lemma 2.7]. Thus $u_{\hat{M}}(\Phi \hat{R})=$ 0 if and only if $\operatorname{Ass}_{\hat{R}} \hat{M}=T(\Phi \hat{R}, \hat{M})$ and the proof is complete.

The next theorem gives a result for representable general formal local cohomology modules.

Theorem 10. Let $(R, \mathfrak{m})$ be a local ring, $\Phi$ a system of ideals of $R$ and $M$ a finitely generated $R$-module. Let $i$ be an integer such that $\mathfrak{F}_{\Phi}^{i}(M)$ is nonzero and representable. Then there exists an ideal $\mathfrak{a} \in \Phi$ such that $\mathfrak{a} \subseteq \mathfrak{p}$ for all $\mathfrak{p} \in \operatorname{Att}_{R} \mathfrak{F}_{\Phi}^{i}(M)$

Proof. Let $\mathfrak{F}_{\Phi}^{i}(M)=S_{1}+S_{2}+\ldots+S_{n}$ be a minimal secondary representation of $\mathfrak{F}_{\Phi}^{i}(M)$ where $S_{j}$ is non-zero and $\mathfrak{p}_{j}$-Secondary for $j=1,2, \ldots, n$.

Let $1 \leqslant j \leqslant n$. Since $S_{j} \neq 0$, there exists $0 \neq a=\left(a_{i}\right) \in S_{j} \subseteq$ $\mathfrak{F}_{\Phi}^{i}(M)=\lim _{\mathfrak{a} \in \Phi} \mathrm{H}_{\mathfrak{m}}^{i}(M / \mathfrak{a} M)$.

Let $a_{k}$ be the first nonzero component of $a$. Thus there exists an ideal $\mathfrak{a}_{k} \in \Phi$ such that $a_{k} \in \mathrm{H}_{\mathfrak{m}}^{i}\left(M / \mathfrak{a}_{k} M\right)$. We claim $\mathfrak{a}_{k} \subseteq \mathfrak{p}_{j}$. If not, then there exists $u \in \mathfrak{a}_{k} \backslash \mathfrak{p}_{j}$. Since $u \notin \mathfrak{p}_{j}$, we have $u S_{j}=S_{j}$. Thus $a \in S_{j}=u S_{j} \subseteq u \mathfrak{F}_{\Phi}^{i}(M)$ But $u \mathrm{H}_{\mathfrak{m}}^{i}\left(M / \mathfrak{a}_{k} M\right)=0$ and so the $k$-th component of each element of $u \mathfrak{F}_{\Phi}^{i}(M)$ is zero. But $a \in u \mathfrak{F}_{\Phi}^{i}(M)$ and the $k$-th component of $a$ is not zero. It follows that $\mathfrak{a}_{k} \subseteq \mathfrak{p}_{j}$ where $\mathfrak{a}_{k} \in \Phi$. Hence, we proved that for each integer $j \in\{1, \ldots, n\}$ there exists an ideal $\mathfrak{b}_{j} \in \Phi$ such that $\mathfrak{b}_{j} \subseteq \mathfrak{p}_{j}$. Since $\Phi$ is a system of ideals there exists an ideal $\mathfrak{a} \in \Phi$ such that $\mathfrak{a} \subseteq \mathfrak{b}_{1} \mathfrak{b}_{2} \cdots \mathfrak{b}_{n} \subseteq \mathfrak{p}_{j}$ for all $j \in\{1, \ldots, n\}$, this completes the proof. 
Corollary 3. Let $(R, \mathfrak{m})$ be a local ring, $\Phi$ a system of ideals of $R$ and $M$ a finitely generated $R$-module. Let $i$ be an integer such that $\mathfrak{F}_{\Phi}^{i}(M)$ is nonzero and representable. Then there exists an ideal $\mathfrak{a} \in \Phi$ such that $\mathfrak{a} \mathfrak{F}_{\Phi}^{i}(M)=0$.

Proof. By [5, 7.2.11] $\bigcap_{\mathfrak{p} \in \operatorname{Att} \mathfrak{F}_{\Phi}^{i}(M)} \mathfrak{p}=\sqrt{\left(0: \mathfrak{F}_{\Phi}^{i}(M)\right)}$. Thus by Theorem 10 we conclude that there exists an ideal $\mathfrak{b}$ in $\Phi$ and an integer $n$ such that, $\mathfrak{b}^{n} \mathfrak{F}_{\Phi}^{i}(M)=0$. Since $\Phi$ is a system of ideals, there exists an ideal $\mathfrak{a}$ in $\Phi$ such that $\mathfrak{a} \subseteq \mathfrak{b}^{n}$. Therefore $\mathfrak{a} \mathfrak{F}_{\Phi}^{i}(M)=0$, as desired.

Let $R$ be a ring, $\Phi$ a system of ideals of $R$ and $M$ an $R$-module. Recall that

$$
\Gamma_{\Phi}(M):=\{x \in M: \mathfrak{a} x=0 \text { for some } \mathfrak{a} \text { in } \Phi\} .
$$

We say that $M$ is $\Phi$-torsion if $M=\Gamma_{\Phi}(M)$ and that $M$ is $\Phi$-torsion-free if $\Gamma_{\Phi}(M)=0$. For a finitely generated $R$-module $M$, it is easy to see that $M$ is $\Phi$-torsion-free if and only if, for each $\mathfrak{a} \in \Phi, \mathfrak{a}$ contains a non-zero-divisor on $M$.

In order to state the next result we recall the concept of Matlis dual. Let $M$ be an $R$-module and $E(R / \mathfrak{m})$ the injective envelope of $R / \mathfrak{m}$. The module $\mathrm{D}(M)=\operatorname{Hom}_{R}(M, E(R / \mathfrak{m}))$ is called the Matlis dual of $M$.

Lemma 1. Let $(R, \mathfrak{m})$ be a complete local ring, $\Phi$ a system of ideals of $R$ and $M$ a finitely generated $R$-module. Then

(i) $M$ is $\Phi$-adically complete (i.e $M \simeq \lim _{\mathfrak{a} \in \Phi}(M / \mathfrak{a} M)$ ),

ii) $\mathfrak{F}_{\Phi}^{0}(M)$ is finitely generated $R$-module.

Proof. i) Since $M$ is finitely generated, $\mathrm{D}(M)$ is Artinian and so $\mathrm{D}(M)$ is $\mathfrak{m}$-torsion. For each $i \in \mathbb{N}$, there exists $\mathfrak{a} \in \Phi$ such that $\mathfrak{a} \subseteq \mathfrak{m}^{i}$. Hence $\mathrm{D}(M)$ is $\Phi$-torsion and we have

$$
\mathrm{D}(M)=\bigcup_{\mathfrak{a} \in \Phi}(0: \mathrm{D}(M) \mathfrak{a}) \simeq \underset{\mathfrak{a} \in \Phi}{\lim _{\longrightarrow}} \operatorname{Hom}_{R}(R / \mathfrak{a}, \mathrm{D}(M)) .
$$

Thus

$$
\begin{aligned}
M & \simeq \mathrm{DD}(M) \simeq \mathrm{D}\left(\underset{\mathfrak{a} \in \Phi}{\lim _{\longrightarrow}} \operatorname{Hom}_{R}(R / \mathfrak{a}, \mathrm{D}(M))\right) \simeq \\
& \simeq \lim _{\mathfrak{a} \in \Phi} R / \mathfrak{a} \otimes_{R} \mathrm{DD}(M) \simeq \lim _{\mathfrak{a} \in \Phi} M / \mathfrak{a} M .
\end{aligned}
$$

ii) By definition $\mathfrak{F}_{\Phi}^{0}(M)=\lim _{\mathfrak{a} \in \Phi} H_{\mathfrak{m}}^{0}(M / \mathfrak{a} M)$. Since $\mathrm{H}_{\mathfrak{m}}^{0}(M / \mathfrak{a} M) \subseteq$ $M / \mathfrak{a} M$ for all $\mathfrak{a} \in \Phi$, by (i) we get

$$
\mathfrak{F}_{\Phi}^{0}(M) \subseteq \lim _{\mathfrak{a} \in \Phi}(M / \mathfrak{a} M) \simeq M .
$$


Since $M$ is finitely generated we conclude that $\mathfrak{F}_{\Phi}^{0}(M)$ is finitely generated, as required.

Lemma 2. Let $\Phi$ be a system of ideals of $R$ and $L \stackrel{f}{\rightarrow} M \stackrel{g}{\rightarrow} N$ be a exact sequence of $R$-modules and $R$-homomorphisms. Suppose that there exist two ideal $\mathfrak{a}$ and $\mathfrak{b}$ in $\Phi$ such that $\mathfrak{a} L=0$ and $\mathfrak{b} N=0$. Then there exists an ideal $\mathfrak{c} \in \Phi$ such that $\mathfrak{c} M=0$.

Proof. Since $\mathfrak{b} g(M)=0$, we have $\mathfrak{b} M \subseteq \operatorname{ker} g=\operatorname{im} f$. But $\mathfrak{a} L=0$, and so $\mathfrak{a}(\operatorname{im} f)=0$. Thus $\mathfrak{a} \mathfrak{b} M=0$. But, there exists an ideal $\mathfrak{c} \in \Phi$ such that $\mathfrak{c} \subseteq \mathfrak{a} \mathfrak{b}$. Therefore $\mathfrak{c} M=0$ and the proof is complete.

For the following proof we need the next Lemma.

Lemma 3. Let $(R, \mathfrak{m})$ be a local ring, $\Phi$ a system of ideals of $R$ and $M$ a finitely generated $R$-module. Let $M$ be an $\Phi$-torsion $R$-module. Then $\mathfrak{F}_{\Phi}^{i}(M) \cong \mathrm{H}_{\mathfrak{m}}^{i}(M)$. Therefore $\mathfrak{F}_{\Phi}^{i}(M)$ is Artinian for all $i \geqslant 0$.

Proof. It is easy to see that, since $M$ is finitely generated and $\Phi$-torsion there exists an ideal $\mathfrak{a}$ in $\Phi$ such that $\mathfrak{a} M=0$. We put $\Psi=\{\mathfrak{b} \in \Phi \mid \mathfrak{b} \subseteq \mathfrak{a}\}$. Then $\Psi$ is cofinal in $\Phi$. Thus we may assume that $\mathfrak{b} \subseteq \mathfrak{a}$ for all $\mathfrak{b} \in \Phi$ and so $\mathfrak{b} M=0$ for all $\mathfrak{b} \in \Phi$. Hence

$$
\mathfrak{F}_{\Phi}^{i}(M) \cong \lim _{\mathfrak{b} \in \Phi} \mathrm{H}_{\mathfrak{m}}^{i}(M / \mathfrak{b} M) \cong \lim _{\mathfrak{b} \in \Phi} \mathrm{H}_{\mathfrak{m}}^{i}(M) \cong \mathrm{H}_{\mathfrak{m}}^{i}(M)
$$

for all $i \geqslant 0$, as desired.

Theorem 11. Let $(R, \mathfrak{m})$ be a local ring, $\Phi$ a system of ideals of $R$ and $M$ a finitely generated $R$-module. Let $t \in \mathbb{N}$. Then the following statements are equivalent:

(i) $\mathfrak{F}_{\Phi}^{i}(M)$ is Artinian for all $i<t$,

(ii) $\mathfrak{F}_{\Phi}^{i}(M)$ is representable for all $i<t$,

(iii) there exists an ideal $\mathfrak{a}$ in $\Phi$ such that, $\mathfrak{a} \mathfrak{F}_{\Phi}^{i}(M)=0$ for all $i<t$.

Proof. (i) $\Rightarrow$ (ii); Any Artinian $R$-module is representable.

(ii) $\Rightarrow$ (iii): By Corollary 3 .

(iii) $\Rightarrow(\mathrm{i})$ : We use induction on $t$. Since $\mathfrak{F}_{\Phi}^{i}(M) \simeq \mathfrak{F}_{\Phi \widehat{R}}^{i}(\widehat{M})$ by Theorem 4 , we may assume that $R$ is complete. Let $t=1$. By Lemma 1(ii), $\mathfrak{F}_{\Phi}^{0}(M)$ is a finitely generated $R$-module. By assumption $\operatorname{Supp}_{R}\left(\mathfrak{F}_{\Phi}^{0}(M)\right) \subseteq$ $V(\mathfrak{a})$ and so by Corollary 1 we conclude that $\operatorname{Supp}_{R}\left(\mathfrak{F}_{\Phi}^{0}(M)\right) \subseteq V(\mathfrak{m})$. Thus $\mathfrak{F}_{\mathfrak{a}}^{0}(M)$ is Artinian. 
Now suppose, inductively, that $t>0$ and $\mathfrak{F}_{\Phi}^{i}(M)$ is Artinian for all $i \leqslant t-2$. We show that $\mathfrak{F}_{\mathfrak{a}}^{t-1}(M)$ is Artinian. By Theorem 6 , the short exact sequence

$$
0 \longrightarrow \Gamma_{\Phi}(M) \longrightarrow M \longrightarrow M / \Gamma_{\Phi}(M) \longrightarrow 0
$$

implies the long exact sequence

$$
\begin{aligned}
\cdots & \longrightarrow \mathfrak{F}_{\Phi}^{i-1}\left(\Gamma_{\Phi}(M)\right) \longrightarrow \mathfrak{F}_{\Phi}^{i-1}(M) \\
& \longrightarrow \mathfrak{F}_{\Phi}^{i-1}\left(M / \Gamma_{\Phi}(M)\right) \longrightarrow \mathfrak{F}_{\Phi}^{i}\left(\Gamma_{\Phi}(M)\right) \longrightarrow \cdots .
\end{aligned}
$$

But $\mathfrak{F}_{\Phi}^{i}\left(\Gamma_{\Phi}(M)\right)$ is Artinian for all $i$ by Lemma 3. Thus by using the above long exact sequence it follows that $\mathfrak{F}_{\Phi}^{i}(M)$ is Artinian if and only if $\mathfrak{F}_{\Phi}^{i}\left(M / \Gamma_{\Phi}(M)\right)$ is Artinian for all $i$. On the other hand, since $\Phi$ is a system of ideals, by Corollary 3 we can find an ideal $\mathfrak{b} \in \Phi$ such that $\mathfrak{b} \mathfrak{F}_{\Phi}^{i}\left(\Gamma_{\Phi}(M)\right)=0$ for all $i \leqslant t$. By assumption and lemma 2 we conclude that there exists an ideal $\mathfrak{c} \in \Phi$ such that $\mathfrak{c} \mathfrak{F}_{\Phi}^{i}\left(M / \Gamma_{\Phi}(M)\right)=0$ for all $i<t$. Therefore we can and do assume that $M$ is an $\Phi$-torsion-free $R$-module. Since $\mathfrak{a} \in \Phi$, it is easy to see that $\mathfrak{a}$ contains an element $r$ which is a non-zerodivisor on $M$. The short exact sequence

$$
0 \longrightarrow M \stackrel{r}{\longrightarrow} M \longrightarrow M / r M \longrightarrow 0
$$

induces a long exact sequence

$0 \rightarrow \mathfrak{F}_{\Phi}^{0}(M) \stackrel{r}{\rightarrow} \mathfrak{F}_{\Phi}^{0}(M) \rightarrow \cdots \rightarrow \mathfrak{F}_{\Phi}^{i}(M) \stackrel{r}{\rightarrow} \mathfrak{F}_{\Phi}^{i}(M) \rightarrow \mathfrak{F}_{\Phi}^{i}(M / r M) \rightarrow \cdots$

By assumption and the above long exact sequence and lemma 2 , it follows that there exists an ideal $\mathfrak{b} \in \Phi$ such that $\mathfrak{b} \mathfrak{F}_{\Phi}^{i}(M / r M)=0$ for all $i<t-1$. Thus, by the inductive hypothesis, we conclude that $\mathfrak{F}_{\Phi}^{t-2}(M / r M)$ is Artinian. Since $r \mathfrak{F}_{\Phi}^{t-1}(M) \subseteq \mathfrak{a} \mathfrak{F}_{\Phi}^{t-1}(M)=0$, the above long exact sequence implies that $\mathfrak{F}_{\Phi}^{t-2}(M / r M) \longrightarrow \mathfrak{F}_{\Phi}^{t-1}(M) \longrightarrow 0$ is exact. But $\mathfrak{F}_{\Phi}^{t-2}(M / r M)$ is Artinian and so $\mathfrak{F}_{\mathfrak{a}}^{t-1}(M)$ is Artinian, as required.

Theorem 12. Let $(R, \mathfrak{m})$ be a local ring, $\Phi$ a system of ideals of $R$ and $M$ a finitely generated $R$-module. Let $t \in \mathbb{N}$. Then the following statements are equivalent:

(i) $\mathfrak{F}_{\Phi}^{i}(M)$ is Artinian for all $i>t$,

(ii) $\mathfrak{F}_{\Phi}^{i}(M)$ is representable for all $i>t$,

(iii) there exists an ideal $\mathfrak{a}$ in $\Phi$ such that, $\mathfrak{a} \mathfrak{F}_{\Phi}^{i}(M)=0$ for all $i>t$. 
Proof. (i) $\Rightarrow$ (ii): It is clear.

(ii) $\Rightarrow$ (iii): By Corollary 3 .

(iii) $\Rightarrow$ (i): The proof can be easily obtained by extending the proof of [4, Theorem 2.9] mutatis mutandis to this general case.

Let $\mathfrak{a}$ be an ideal of a local $\operatorname{ring}(R, \mathfrak{m})$ and $M$ a finitely generated $R$-module of dimension $d$. By Theorem $8 \mathfrak{F}_{\Phi}^{d}(M)$ is Artinian. In the next result we determine the set $\operatorname{Att}_{R} \mathfrak{F}_{\Phi}^{d}(M)$.

Theorem 13. Let $(R, \mathfrak{m})$ be a local ring, $\Phi$ a system of ideals of $R$ and $M$ a finitely generated $R$-module of dimension $d$. Then there exists an ideal $\mathfrak{a}$ in $\Phi$ such that $\operatorname{Att}_{R} \mathfrak{F}_{\Phi}^{d}(M)=\operatorname{Assh}_{R}(M) \cap \mathrm{V}(\mathfrak{a})$.

Proof. Let $w:=\max \{\operatorname{dim}(M / \mathfrak{a} M): \mathfrak{a} \in \Phi\}$. If $w<d$ then $\mathfrak{F}_{\Phi}^{d}(M)=0$ by Theorem 7 and so there is nothing to prove. Thus we suppose that $w=d$.

By Theorem 10 there exists an ideal $\mathfrak{a} \in \Phi \operatorname{such}$ that $\operatorname{Att}_{R} \mathfrak{F}_{\Phi}^{d}(M) \subseteq$ $\mathrm{V}(\mathfrak{a})$. But by Theorem 8 and [5, 7.3.2] $\operatorname{Att}_{R} \mathfrak{F}_{\Phi}^{d}(M) \subseteq \operatorname{Att}_{R} \mathrm{H}_{\mathfrak{m}}^{d}(M)=$ $\operatorname{Assh}_{R}(M)$. Thus $\operatorname{Att}_{R} \mathfrak{F}_{\mathfrak{a}}^{d}(M) \subseteq \operatorname{Assh}_{R}(M) \cap \mathrm{V}(\mathfrak{a})$.

Conversly, assume that $\mathfrak{a} \in \Phi$. We show that $\operatorname{Assh}_{R}(M) \cap \mathrm{V}(\mathfrak{a}) \subseteq$ $\operatorname{Att}_{R} \mathfrak{F}_{\Phi}^{d}(M)$. Let $\mathfrak{p} \in \operatorname{Assh}_{R}(M) \cap \mathrm{V}(\mathfrak{a})$. By [8, 6.8], there exists a $\mathfrak{p}$-primary submodule $N$ of $M$ such that $\operatorname{Ass}(M / N)=\{\mathfrak{p}\}$ and $\mathfrak{p}=$ $\sqrt{(0:(M / N))}$. Thus $\operatorname{dim} M / N=\operatorname{dim} R / \mathfrak{p}=\operatorname{dim} M$. Since $\mathfrak{a} \subseteq \mathfrak{p}$ we have $\sqrt{\mathfrak{a}} \subseteq \sqrt{(0:(M / N))}$.

Thus we can see that $\operatorname{Supp}_{R}((M / N) / \mathfrak{a}(M / N))=\operatorname{Supp}_{R}(M / N)$ and $\operatorname{dim}((M / N) / \mathfrak{a}(M / N))=\operatorname{dim}(M / N)$. Now by Theorem $7, \mathfrak{F}_{\Phi}^{d}(M / N) \neq 0$. Hence

$$
\phi \neq \operatorname{Att}_{R} \mathfrak{F}_{\Phi}^{d}(M / N) \subseteq \operatorname{Att}_{R} \mathrm{H}_{\mathfrak{m}}^{d}(M / N) \subseteq \operatorname{Ass}(M / N)=\{\mathfrak{p}\}
$$

Therefore we have $\operatorname{Att}_{R} \mathfrak{F}_{\Phi}^{d}(M / N)=\{\mathfrak{p}\}$. But the exact sequence

$$
0 \rightarrow N \rightarrow M \rightarrow M / N \rightarrow 0
$$

induces $\mathfrak{F}_{\Phi}^{d}(M) \rightarrow \mathfrak{F}_{\Phi}^{d}(M / N) \rightarrow 0$. Thus $\{\mathfrak{p}\}=\operatorname{Att}_{R} \mathfrak{F}_{\Phi}^{d}(M / N) \subseteq$ $\operatorname{Att}_{R} \mathfrak{F}_{\Phi}^{d}(M)$. Therefore $\mathfrak{p} \in \operatorname{Att}_{R} \mathfrak{F}_{\Phi}^{d}(M)$. This completes the proof.

Corollary 4. Let $(R, \mathfrak{m})$ be a local ring, $\Phi$ a system of ideals of $R$ and $M$ and $N$ be two finitely generated $R$-modules of dimension $d$ such that $\operatorname{Supp}_{R} M=\operatorname{Supp}_{R} N$. Then $\operatorname{Att}_{R} \mathfrak{F}_{\Phi}^{d}(M)=\operatorname{Att}_{R} \mathfrak{F}_{\Phi}^{d}(N)$. 
Proof. By Theorem 13 there exist two ideals $\mathfrak{a}$ and $\mathfrak{b}$ in $\Phi$ such that $\operatorname{Att}_{R} \mathfrak{F}_{\Phi}^{d}(M)=\operatorname{Assh}_{R} M \cap \mathrm{V}(\mathfrak{a})$ and $\operatorname{Att}_{R} \mathfrak{F}_{\Phi}^{d}(N)=\operatorname{Assh}_{R} N \cap \mathrm{V}(\mathfrak{b})$. But by assumption we have $\operatorname{Assh}_{R} M=\operatorname{Assh}_{R} N$. On the other hand, by using the proof of Theorem 13,

$$
\operatorname{Att}_{R} \mathfrak{F}_{\Phi}^{d}(M)=\operatorname{Assh}_{R} M \cap \mathrm{V}(\mathfrak{a})=\operatorname{Assh}_{R} N \cap \mathrm{V}(\mathfrak{a}) \subseteq \operatorname{Att}_{R} \mathfrak{F}_{\Phi}^{d}(N) .
$$

Similarly $\operatorname{Att}_{R} \mathfrak{F}_{\Phi}^{d}(N) \subseteq \operatorname{Att}_{R} \mathfrak{F}_{\Phi}^{d}(M)$. This completes the proof.

\section{Acknowledgement}

The author thanks the referee for his careful reading and useful suggestions on this paper.

\section{References}

[1] M. Asgharzadeh, K. Divaani-Aazar, Finiteness properties of formal local cohomology modules and Cohen-Macaulayness, Comm. Algebra, N.39, 2011, pp.1082-1103.

[2] M. H. Bijan-Zadeh, Torsion theories and local cohomology over commutative noetherian rings, J. london Math. Soc., N.19, 1979, pp.402-410.

[3] M. H. Bijan-Zadeh, A common generalization of local cohomology theories, Glascow Math. J., N.21, pp.173-181.

[4] M. H. Bijan-Zadeh, Sh. Rezaei, Artinianness and attached primes of formal local cohomology modules, Algebra Colloquium, N.21(2), 2014, pp.307-316.

[5] M. Brodmann, R. Y. Sharp, Local cohomology: an algebraic introduction with geometric applications, Cambridge Univ. Press, N.60, 1998.

[6] M. Eghbali, On Artinianness of formal local cohomology, colocalization and coassociated primes, Math. Scand., N.113(1), 2013, pp.5-19.

[7] T. H. Freitas, V. H. Jorge Pérez, On formal local cohomology modules with respect to a pair of ideals, J. Commut. Algebra, N.3, 2016, pp.337-366.

[8] H. Matsumura, Commutative ring theory, Cambridge Univ. Press, 1986.

[9] Sh. Rezaei, Minimaxness and finiteness properties of formal local cohomology modules, Kodai Math. J., N.38(2), 2015, pp.430-436.

[10] Sh. Rezaei, Some results on top local cohomology and top formal local cohomology modules, Comm. in Alg., N.45(2), 2017, pp.1935-1940.

[11] Sh. Rezaei, A Generalization of formal local cohomology modules, Kyungpook Math. J., N.56, 2016, pp.737-743.

[12] P. Schenzel, On formal local cohomology and connectedness, J. Algebra, N.315(2), 2007, pp.894-923.

[13] P. Schenzel, On the use of local cohomology and geometry, Progress in Math., 1998, pp.241-292.

\section{Shahram Rezaei Payame Noor University, Tehran, Iran E-Mail(s): Sha.Rezaei@gmail.com}

Received by the editors: 23.02.2018

and in final form 28.08.2020. 